\title{
MALDI Imaging MS: Molecular Visualization of Tissues in Drug Discovery and Development
}

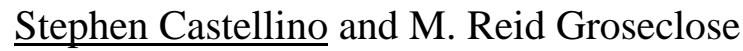

GlaxoSmithKline, Drug Metabolism and Pharmacokinetics, Research Triangle Park, NC, USA

Our current approach to risk assessment in the development of safe and efficacious drugs is primarily based on the parent drug plasma exposure (area under the concentration/time curve; AUC) in both animal models and in humans [1]. The successful implementation of this strategy is driven by a robust analytical methodology: LC-MS/MS. One of the key advantages of this "exposure-centric" approach is ease of translation from animal models to clinical. However, it has been recognized that measuring drug plasma exposure is not ideal given that most targets are not located within the plasma compartment and determining the tissue distribution of not only the parent drug but its metabolites would provide greater insight into our understanding of pharmacology and toxicology.

What has been missing, until recently, is an analytical method capable of determining the tissue distribution of drugs and drug metabolites. Matrix assisted laser desorption/ionization (MALDI) imaging mass spectrometry (IMS) is uniquely suited for this task [2]. MALDI IMS is a highly sensitive technique (attomole), does not require isotopic labeling of analytes, and is capable of high spatial resolution $\left(10 \mu \mathrm{m}\right.$ pixels) and spectral resolution (FTICR; $>10^{4}$ resolving power (FWHM)). Furthermore, there is the opportunity to collect endogenous analyte distributions in the same experiment [3]. This allows one to co-register tissue histology images with the corresponding molecular images and visualize biology and chemistry in an integrated fashion. For MALDI IMS, the translation between animal models and clinical trials is though physiological based pharmacokinetic modeling and an enhanced understanding of biochemical mechanisms.

In our experimental protocol, target tissues are removed at necropsy, flash frozen, and stored at low temperature until cryo-sectioning. Tissue sections (6-12 $\mu \mathrm{m}$ thickness) are thaw-mounted on glass slides prior to the application of the MALDI matrix. It is possible to perform whole rodent body MALDI imaging as well. The matrix is generally a small molecular weight highly conjugated molecule that absorbs the UV light from the laser and initiates desorption and the ionization process prior to detection by the mass analyzer. The matrix is usually applied as a fine aerosol containing a mixture of organic solvent and water. Tissue sections serial to those used for the MALDI IMS experiment are collected for histological staining (Figure 1). As is the case with other mass spectrometry methods, quantification of targeted analytes is possible if standards are available. Methods for quantitative MALDI IMS have been described in the literature [4-6].

Several examples illustrate the importance of understanding tissue distribution and the impact of MALDI IMS in drug development. When unexpected seizures were observed in a clinical Phase IIb study for an NNRTI inhibitor, MALDI IMS experiments with several animal models demonstrated that this adverse event was likely do to species differentiation of metabolite disposition and biotransformation. In this study, the exposure of the parent drug in plasma provided no clues to the mechanistic basis for the seizures in patients [7]. In a similar vein, monitoring the plasma levels of a late stage drug was not fully informing on the risk assessment of testicular toxicity in the rodent. In this case, MALDI IMS data demonstrated the testicular accumulation of long lived metabolites correlated 
with the time course of testicular injury. The risk assessment of testicular toxicity was linked to the biotransformation rather than the parent drug plasma level. In addition, several studies involving nepherotoxicity due to tubular deposits have been investigated using MALDI IMS. In one case, the deposits were determined to contain primarily calcium phosphate, which provided a basis for a mechanistic hypothesis. In the other case, the tubular deposits were shown to consist of several metabolites formed through oxidation pathways. In both instances, the parent drug was not directly linked to the toxicity.

In summary, through the visualization of molecular tissue distributions combined with other imaging modalities, MALDI IMS is dramatically changing the way we discover and develop safe and efficacious drugs. While most of our efforts have focused on understanding drug related adverse events in the late stages of drug development, we have also found increasing demand and application in all stages of drug discovery and development including target engagement and PK/PD studies, (Figure 2).

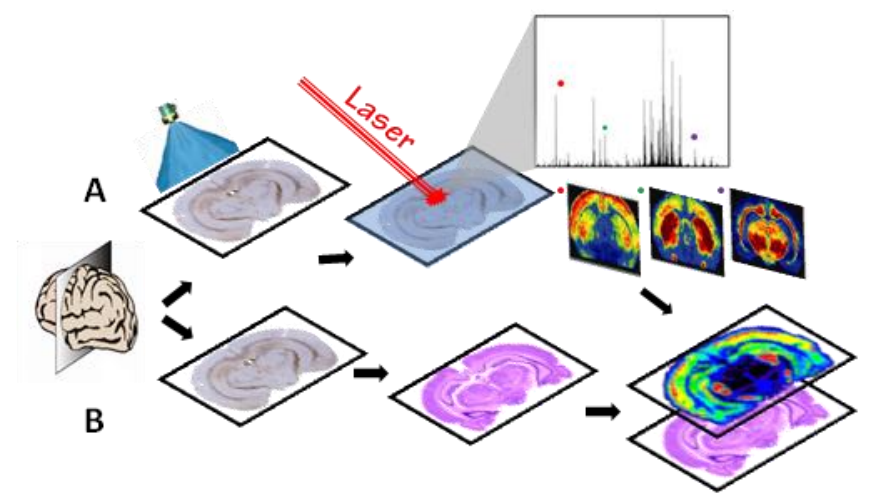

Figure 1. A) MALDI IMS pathway- tissue sectioning, matrix application, data collection, and ion images. B) Serial sectioning, histological staining and/or IHC, co-registration with ion images

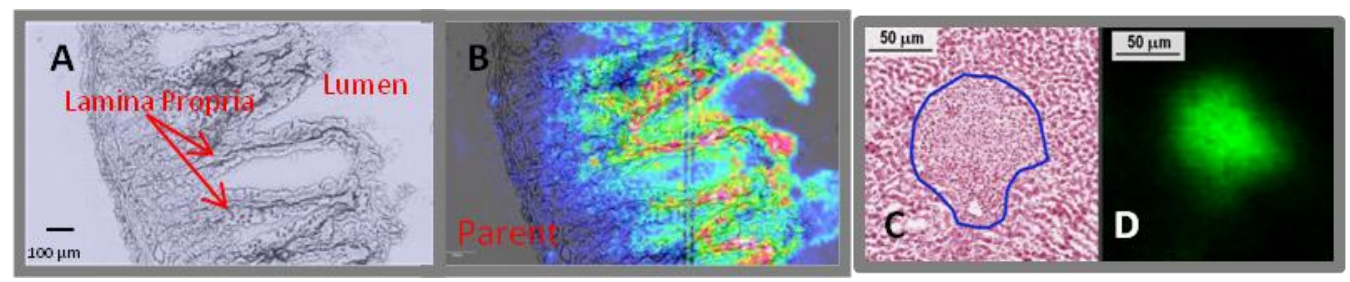

Figure 2. A) Optical Image of rat jejunum B) Ion image of drug from serial section C) H\&E of rat liver with highlighted area of necrosis/inflammation D) Lipid ion image from serial section

References:

[1] P Muller and M Milton, Nature Reviews Drug Discovery (2012), p. 751.

[2] R Caprioli, T Farmer, and J Gile, Anal. Chemistry, 69, (1997), p. 4751.

[3] S Castellino, M Groseclose, and D Wagner, Bioanalysis, 3, (2011), p. 2427.

[4] Reyzer, M, et al., J. Mass Spectrom. 38, (2003), 1081-1092.

[5] Hamm G, et al, J. Proteomics, 16, (2012), p. 4952

[6] Groseclose M, and Castellino, S, Anal. Chemistry, 85, (2013), p. 10099

[7] Castellino, S, et al, Chem. Res. Toxicol., 26, (2013), p. 241 